American Journal of Applied Sciences 5 (7): 755-762, 2008

ISSN 1546-9239

(C) 2008 Science Publications

\title{
Low-Noise High-Accuracy TOF Laser Range Finder
}

\author{
Shahram Mohammad Nejad and Saeed Olyaee \\ Optoelectronic and Laser Laboratory, Iran University of Science and Technology, Narmak, 16846, \\ Tehran, Iran
}

\begin{abstract}
This paper presents a new low-noise high-accuracy laser range finder based on the Time-OfFlight (TOF) method. A Q-switched Nd:YAG laser at $1064 \mathrm{~nm}$ as a source and a SLIK avalanche photodiode as a detector are used. The optical section of the system including $\mathrm{ZnS} / \mathrm{MgF}_{2}$ optical filter, designed by $\mathrm{C}^{++}$and Zemax software, has been successfully implemented and tested. The quality factor of the optical filter is obtained about 2.53. Using the 1.5 MW Nd:YAG laser, the lowest detectable optical power is limited to about $8.14 \mathrm{nW}$. The absolute range finding error in the range of $0.3-20 \mathrm{~km}$ is also measured as $\delta_{\mathrm{r}}= \pm 50 \mathrm{~cm}$. Furthermore, the sampling rate of the distance measurement can be adjusted between 1 and 20 samples per second.
\end{abstract}

Keywords: Time-of-flight, laser range finder, Q-switched Nd:YAG laser, optical filters

\section{INTRODUCTION}

Time-of-flight method is the most widely used technique for long distance measurement over length scales of several hundred meters to tens of kilometers. Continuous wave lasers are usually being used in the interferometry, triangulation, phase-shift and frequency modulation continuous wave (FMCW) techniques. As a result, these techniques are being used for lower range finding processes. The interferometry technique is generally used for short distances and it has a very high accuracy $^{[1-4]}$. The phase shift technique is usually used to measure the intermediate distances up to several hundred meters ${ }^{[5]}$. FMCW or the combination of interferometry and phase shift techniques could also being used to measure the intermediate distances with high accuracy ${ }^{[6-11]}$.

Because of using the continuous wave $(\mathrm{CW})$ or burst lasers, the related techniques could not be used to measure distances above $1 \mathrm{~km}$. As the repetition rate of the transmitter power increases, the range finding system become complicated and a cooling system is needed. On the other hand, if the repetition rate decreases, the detection of high-speed targets becomes very difficult. Therefore, it is necessary to trade-off between the maximum distance to be measured, the repetition rate and the system's complication.

\section{MATERIALS AND METHODS}

Principals of the TOF laser range finder: In a TOF laser range finder system, the round-trip time of a short powerful laser pulse is measured from which the distance is determined ${ }^{[12-15]}$. By reducing the pulse width of the signal, the output power of the laser could be increased to several MW, and the signal to noise ratio could also be increased considerably.

Most important sections of the designed TOF system are:

a. The transmitter section including the Q-switched trigger, the HV source to drive the flash tube, the repetition rate controlling, the cooling system and the transmitter optics.

b. The receiver analog section including the avalanche photodiode (APD) receiver, the low-noise and wide-band preamplifier, the converter, the limiter and the receiver optics.

c. The receiver digital section including the isolating circuit, the processing and the interface circuits.

As shown in Fig.1, the transmitter output beam is directed toward the target and its reflection is collected by the receiver optics. Since the laser beam travels a distance of $2 r$, the receiving power is usually very small. The relation between the received power at the optic section, $P_{i}$, and the distance $\mathrm{r}$, is defined as:

Corresponding Author: $\quad$ Saeed Olyaee, Department of Electrical Engineering, Iran University of Science and Technology, Narmak, 16846, Tehran, Iran, Tel: +98 21 77808022, Fax: +98 2177454055 


$$
P_{i}=\frac{\tau \cdot A \cdot \exp (-2 \alpha r)}{\pi r^{2}} . \rho \cdot P_{o p t} \quad, \quad \tau=\tau_{c} \cdot \tau_{o p t}
$$

where $\tau, \alpha$ and $\rho$ are the transmission coefficient (equal to the multiplication of transmission coefficient of the optics and the transmission coefficient of the media), the absorption coefficient and the reflection coefficient of the target ( $\rho=1$ for a perfect mirror and is equal to zero for a black body), respectively. Also $P_{o p t}$ is the laser output power and $A$ is the receiver optic area. Considering 1\% target reflection and $75 \%$ transmission of the media, and assuming a perfectly round optical lens of $5 \mathrm{~cm}$ radius, in a maximum distance of $20 \mathrm{~km}$, the received power will be $70.3 \mathrm{nW}$.

According to Fig.1, a START signal is simultaneously introduced to the processing circuit through a PIN photodiode. By receiving the START signal, a counter is activated until the STOP signal is received. After traveling a distance of $2 r$, the laser beam through the optics arrives on the APD having high responsivity and short rise time. In order to stop the counter, the APD output photocurrent is converted into a voltage signal and then is amplified. The distance between the target and the transmitter in terms of clock pulse frequency $\left(f_{c l k}\right)$ is given by:

$$
r=\frac{c N}{2 f_{c l k}}
$$

where $\mathrm{N}$ is the counted digits (between the START and STOP signals) and $\mathrm{c}$ is the speed of light. In accordance to Eq. 2, the resolution of the system related to the clock pulse is,

$$
\delta_{r}=\frac{C}{2 f_{c l k}}
$$

Design and implementation of the TOF laser range finder: The main function of the transmitting section is to produce a relatively powerful short period pulse, adjust its repetition rate and also produce the START signal. The transmitter circuit schematic is shown in Fig.2. The transmitting system includes the laser (Nd:YAG laser media, polarizer, Q-switched trigger, cavity mirrors, flash lamp and a low-dark current PIN photodiode), the $4.5 \mathrm{KV}$ power supply, the driver and repetition rate generator and the laser cooling system.

Using the Q-switched trigger and the related driving and oscillating circuits, the repetition rate is adjusted in the range of $1-20 \mathrm{~Hz}$. On the other hand, the laser output pulse variation is adjusted to the range of 10-15 ns, which in turn, causes the laser cavity output power to vary. In accordance to the measured $15 \mathrm{~mJ}$ energy, the output power could be adjusted from 1.0 to 1.5 MW.

The Nd:YAG laser was pumped by a Flash Tube. For the flash tube excitation a $4.5 \mathrm{kV}$ power supply is used. Since the power generation of cavity is high, the laser cavity needs to be cooled by an especial cooling system. The starting signal is produced by a silicon PIN photodiode having dark current $I_{d}=1 n A$ and a rise time of $\tau_{r}=5 n s$.

When the Q-switch is triggered and the laser pulse is directed out of the cavity, a current pulse is produced by the PIN photodiode and implied to the processing section to start the counting.

A small fraction of the main reflected beam by the target is received by the receiver section. Hence the START and STOP signals is produced to measure the time-of-flight of the beam. The range finder receiver consists of two analog and digital sections. Figure 3 shows the designed and assembled block diagram of the analog section. As shown in Fig.3, the reflected beam is being detected by an APD. The output signal is amplified by a low-noise and wide-band preamplifier having an input impedance of $50 \Omega$. The amplifier output signal is given by:

$$
V_{o}=P_{i} \cdot \Re \cdot Z_{t} \cdot A_{v}
$$

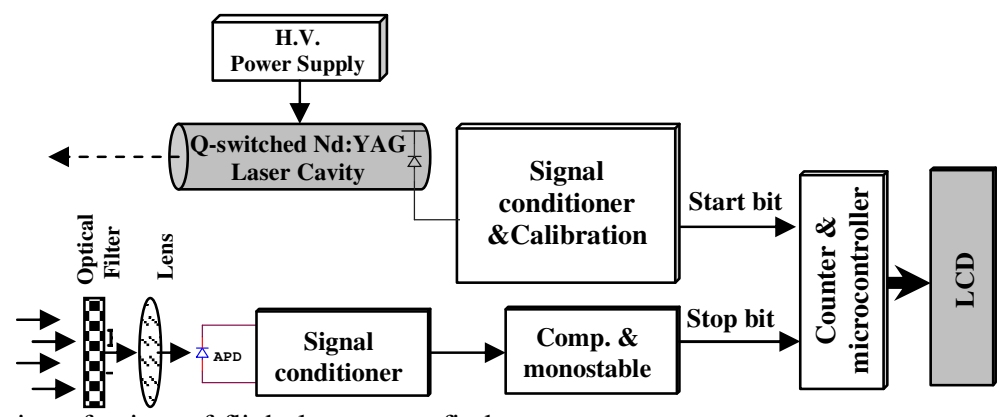

Fig. 1: Schematic representation of a time-of-flight laser range finder 
where $\Re$ is the responsivity of APD, $Z_{t}$ and $A_{v}$ are the trans-impedance gain of the preamplifier and the voltage gain of amplifier, respectively.

The amplifier output is implied to a comparator to allow the increase of the output to the desirable level. The signal comparison level due to the minimum received power $(10 \mathrm{nW})$ at the end of preamplifier is considered to be $14 \mathrm{mV}$. This in turn will reduce the system's noise effects. The pulse width of the signal is then increased by a mono-stable and the STOP signal of the counter is fed to the opto-coupler. So it is implied to the digital section of the system

\section{RESULTS AND DISCUSSION}

Figure 4a represents the preamplifier circuit of the receiver. The APD responsivity is $28 \mathrm{~A} / \mathrm{W}$ at $1064 \mathrm{~nm}$ wavelength, temperature of $23^{\circ} \mathrm{C}$ and reverse bias equal to $327 \mathrm{~V}$. The produced photocurrent is about 280 $\mathrm{nA}$ due to the $10 \mathrm{nW}$ minimum optical power. In addition, the trans-impedance gain of the preamplifier is equal to $Z_{t}=50 \mathrm{k} \Omega$. Figure $4 \mathrm{~b}$ shows the schematics of the power supply circuit for APD biasing.

Furthermore, it is required that the preamplifier gain to be proportional to the received optical power. This can be accomplished by variation of the APD responsivity with respect to the biasing voltage. In the selected avalanche photodiode, the variation of the bias in the range of $320-351 \mathrm{~V}\left(T=23.1^{\circ} C\right)$ causes the responsivity to vary between $18.5 \mathrm{~A} / \mathrm{W}$ to 123.1 $\mathrm{A} / \mathrm{W}^{[16]}$. Therefore the total gain is given by

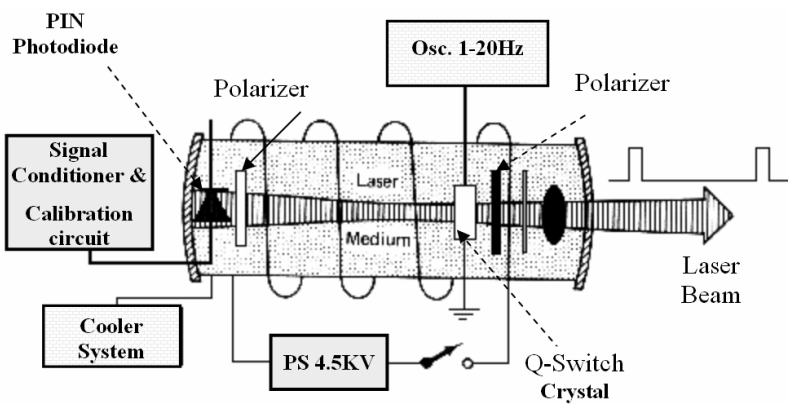

Fig. 2: The designed schematic representation of the transmitter circuit

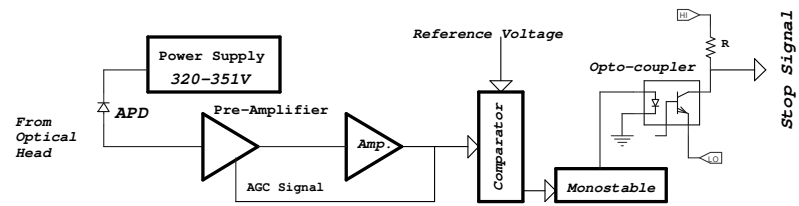

Fig. 3: The schematic representation of the analogue section of the receiver

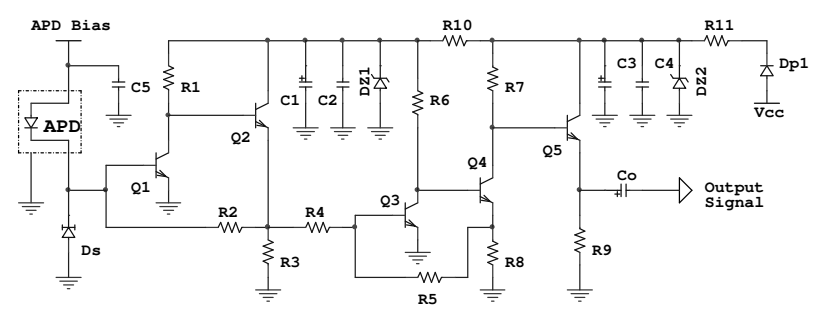

(a)

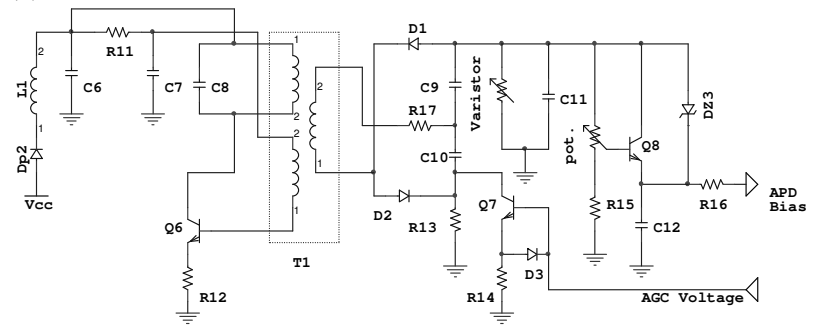

(b)

Fig. 4: Circuitry of (a) The TIA preamplifier and (b) The APD power supply

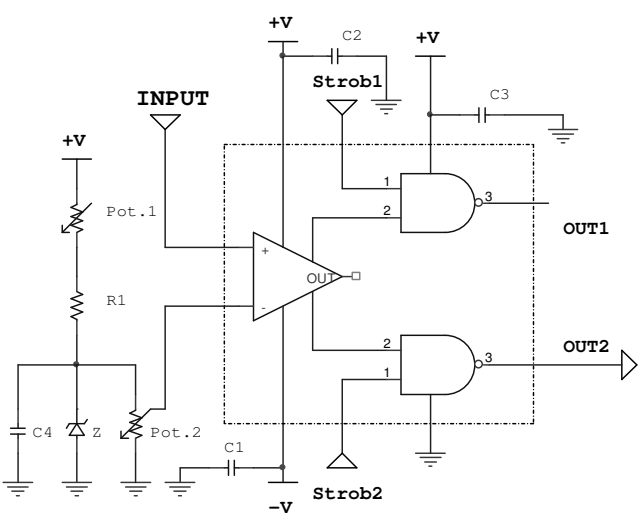

Fig. 5: The comparator circuit

$$
A_{T}=\frac{V_{o}}{P_{i}}=\mathfrak{R} \cdot Z_{t}
$$

As a result, the total gain will vary in the range of $925 \mathrm{kV} / \mathrm{W}$ to $6.155 \mathrm{MV} / \mathrm{W}$. At the end of amplifier, a comparator has been used (Fig.5). As shown in Fig.5, the reference voltage is considered to be $60 \mathrm{mV}$. Hence the noise level of less than $60 \mathrm{mV}$ (according to $10 \mathrm{nW}$ optical power) can not change the comparator output. By using a proper shielding and grounding and reducing the ground resistivity, the optical powers less than $10 \mathrm{nW}$ can also be detected.

Also the responsivity variation as a function of temperature can be canceled by using TEC controller (Fig.6a). The thermistor resistance as a function of temperature is given by ${ }^{[17]}$ : 


$$
T=\left[\frac{1}{3940} \ln \left(10^{-4} R_{T}\right)+\frac{1}{298}\right]^{-1}
$$

At $0^{\circ} \mathrm{C}$, thermistor resistance will be up to $33.5 \mathrm{k} \Omega$ and we will have:

$$
R_{3}+R_{4}=\frac{R_{T} \cdot R_{2}}{R_{1}} \quad, \quad R_{4}=560 \Omega
$$

According to the Fig.6b, the linear region is between -0.14 to $-1.36 \mathrm{~V}$. Therefore by changing the thermistor resistance between 11.62 to $30.36 \mathrm{k} \Omega$, one can adjust temperature from $2^{\circ} \mathrm{C}$ to $21^{\circ} \mathrm{C}$.

A $300 \mathrm{MHz}$ counter is used to measure the roundtrip time of the beam (Fig.7) which results $50 \mathrm{~cm}$ resolution of distance measurement. The obtained data from the counter is sent to the micro-controller and according to this data and the ERROR and PAUSE signals, the proper phrase is presented on the LCD. If for any reasons, the one cycle output signal of the laser was received by the detector, an ERROR signal will be produced. The lack of receiving signal could have many reasons such as the target is located out of range, the beam is being greatly absorbed by the transmitting media, the target being a very absorbing object and so on. With the implementation of the received signal period, the mono-stable output stays low for the least repetition of $1 \mathrm{~Hz}$. Otherwise, the mono-stable output returns to the High condition. As is shown in Fig.7, a selector is utilized to estimate the desired range for the user. This will greatly eliminate the possible mistakes, which may happen in finding the range.

Since the delay time related to the electronic circuits in the START and STOP paths are not equal, it is necessary that this time difference which causes some deviations in the measurements to be eliminated. The time difference could be eliminated either by hardware or software. It should be noted that the counter employed to measure the round-trip time of the laser beam, acts upon the rising edge of the pulse and hence, in the calibration circuit, the High width of the START pulse is changed. This in turn will displace the falling edge of the START signal and changes the time interval between the START and the STOP signals.

The noise consideration in the range finding paths: Detecting the minimum receiving power is also related to the system's noise. The circuit output noise (optical) can be greatly eliminated by designing and implementing proper optics for the system. The main internal noise is the one created in the receiver STOP channel. Due to the isolation of the analogue section from other parts, the noise effect of the other parts is being curtailed. The switching noise of the $4.5 \mathrm{kV}$ flash lamp of the laser is being eliminated by electromagnetic interference (EMI) filters.

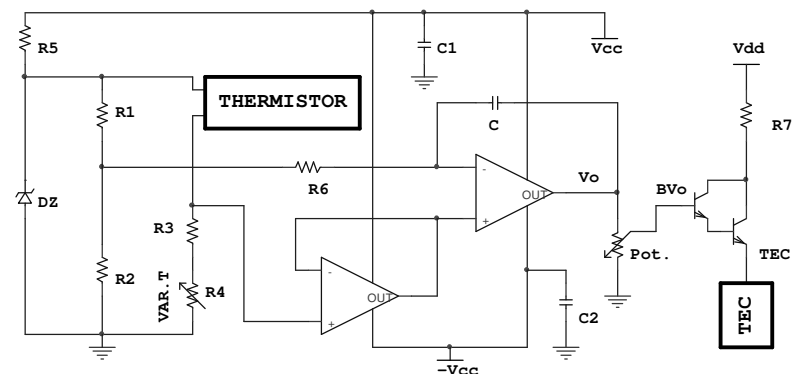

(a)

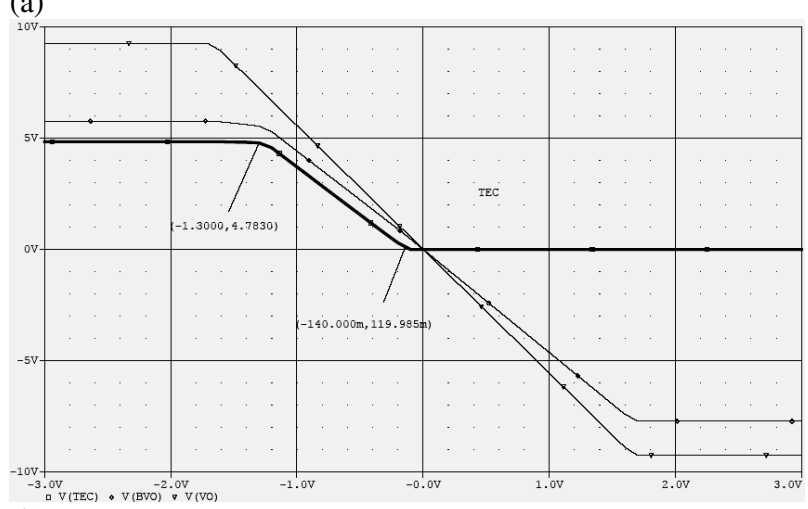

(b)

Fig. 6: (a) The TEC circuit and (b) the simulation result

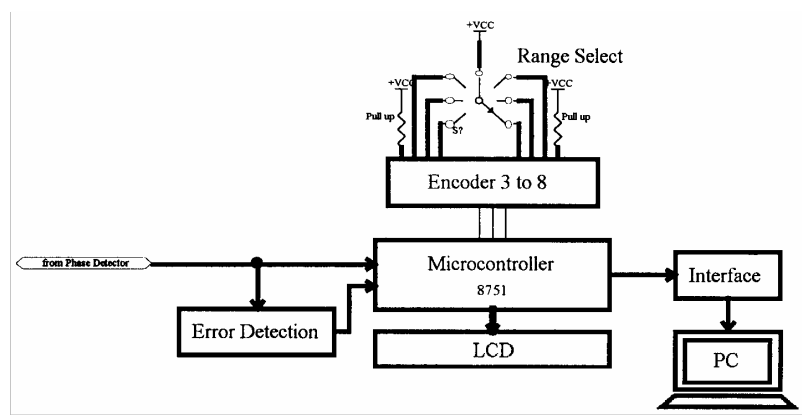

Fig. 7: The block diagram of the receiver digital section

The noise related to the digital section is eliminated by separating the source and the ground from the analogue section of the receiver. However, the main noise related to the receiver channel is the noise of the preamplifier, which is given by ${ }^{[5]}$ :

$$
\left\langle i_{n}{ }^{2}\right\rangle=\frac{4 \kappa T}{Z_{i}}\left(\frac{\pi}{2} \Delta f_{i}\right)
$$

where $\kappa$ is the Boltzmann constant, $T$ is the absolute temperature and $\Delta f_{i}$ is the system bandwidth (100 
MHz). Considering the input impedance of the preamplifier to be equal to $50 \Omega$, the input current noise is $228 \mathrm{nA}$. The equivalent optical power will then become as:

$$
\mathfrak{I}=\frac{\left\langle i_{n}\right\rangle}{\mathfrak{R}}
$$

The 28 A/W responsivity of the APD results the minimum detecting power as much as $8.14 \mathrm{nW}$.

Obviously, the signal-to-noise ratio $(\mathrm{S} / \mathrm{N})$ is one of the main elements determining the resolution of the system. In the designed system, the relation between the resolution and the S/N could be presented by:

$$
\delta_{d}=\frac{C}{2} \cdot \frac{\langle n\rangle}{d U / d t}=\frac{0.35 C}{2 B \cdot S N R}
$$

where $d U / d t$ is the slope of the time dependent pulse and $\langle n\rangle$ is the rms noise value. For repetition rate of $\mathrm{N}$ $(1<\mathrm{N}<20)$, Eq.10 can be rewritten as:

$$
\delta_{d}=\frac{0.35 C}{2 \sqrt{N} \cdot B \cdot S N R}
$$

Considering the SNR of the START path, Eq.11 is corrected as:

$$
\delta_{d}=\frac{0.35 C}{2 \sqrt{N} \cdot B}\left[\frac{1}{\left(S N R_{\text {START }}\right)^{2}}+\frac{1}{\left(S N R_{\text {STOP }}\right)^{2}}\right]^{1 / 2}
$$

Figure 8 shows the resolution in terms of signal to noise ratio. Considering the $50 \mathrm{~cm}$ resolution, the minimum required signal to noise ratio obtained for the worst case ( $\mathrm{N}=1)$ from Eq.11 becomes 0.1. However, in practical situation, the $S N R_{\text {START }}$ is larger and hence the total resolution will be limited to that of the digital section. On the other hand, the signal to noise ratio could be presented as ${ }^{[16]}$ :

$$
S N R=\frac{i_{S}}{i_{n}}=\frac{M \Re P_{\text {inc }}}{\left[2 q\left(k_{T P} P_{\text {inc }} \Re M^{2} F(M)+i_{n a}^{2}\right)\right]^{\frac{1}{2}}}
$$

where $k_{T P}=0.5, M$ is the internal gain of the avalanche photodiode and $F(M)$ is the excess noise factor. The excess noise factor in terms of the effective ionization ratio can be given by:

$$
F(M)=M\left[1-\left(1-k_{\text {eff }}\right)\left(\frac{M-1}{M}\right)^{2}\right]
$$

Figure 9 presents the excess noise factor variation in terms of $M$ for different effective ionization ratios $\left(k_{\text {eff }}\right)$. When effective ionization ratio is decreased, the noise factor will be considerably increased. Using a SLIK structure for avalanche photodiode instead of reach-through and beveled-edge APDs, the $k_{\text {eff }}$ can be decreased up to $0.002^{[18]}$. On the other hand, the excess noise factor increases with $M$. Figure 10 shows the signal to noise ratio in terms of $M$, which for $\mathrm{M}=7.9$ the maximum SNR is obtained.

Simulation of the optical system: The optical part is one of the important sections in a range finding system. A proper optics not only collects and focuses the incident beam, but also it could be designed to filter and eliminate the undesired radiation arriving at the receiver input. The designed optical head of the system is constructed as shown in Fig.11. The reflected beam from the target is directed to the detector by the lens and an optical filter.

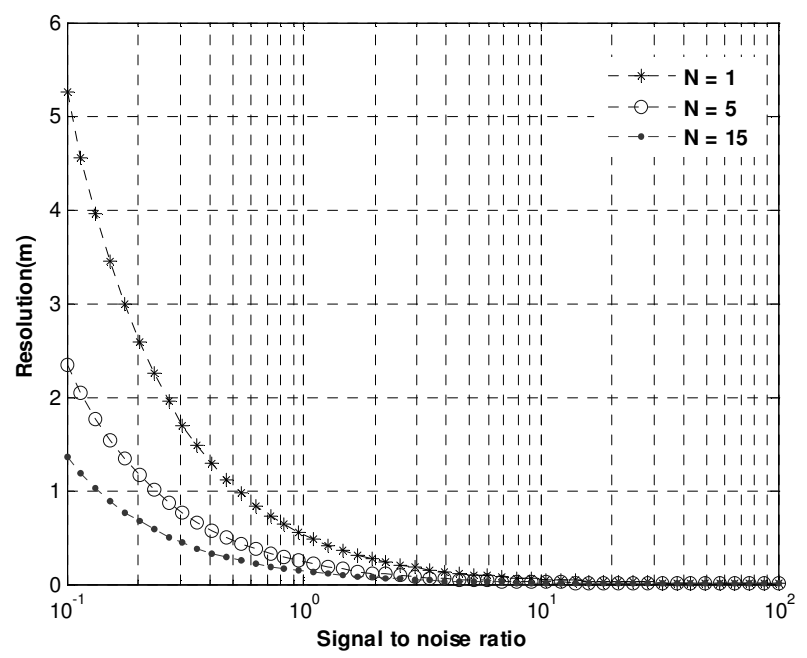

Fig. 8: The resolution in terms of the signal to noise ratio

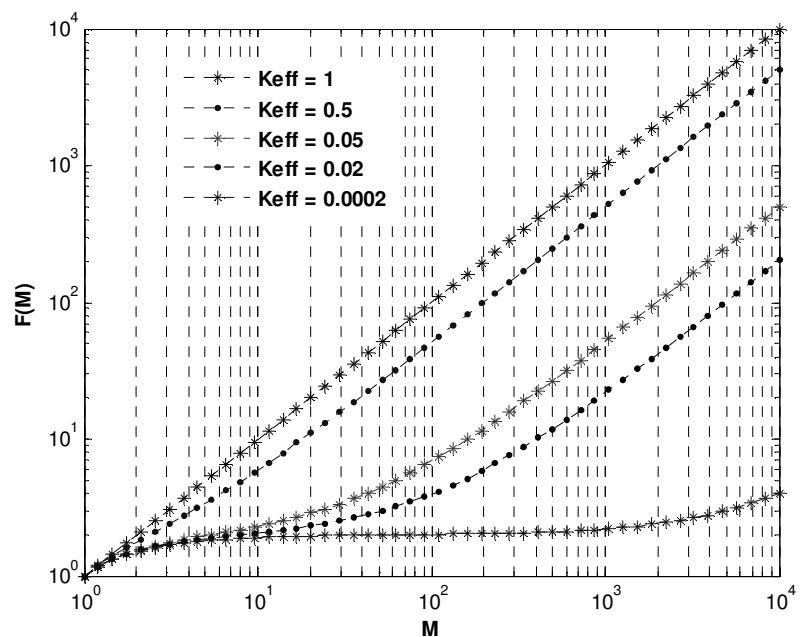

Fig. 9: The excess noise factor variation in terms of $M$ for different effective ionization ratio 
The lens is made of a crown glass having an absorbing range of $10 \mu \mathrm{m}$ and is covered with an antireflection layer of $\mathrm{MgF}_{2}$ with 1.38 reflectivity. The thickness of the anti-reflection layer is $d=1 \mu \mathrm{m} / 4 n=0.18 \mu \mathrm{m}$. This layer is not only to optimize the detectivity but also acts as a proper optical filter. The reason for using the above mentioned glass is that it is not soluble in water, it has the shortest $(1 \mu \mathrm{m})$ passing stripe and is a very good absorbent of the wavelengths above $3.5 \mu \mathrm{m}$ (the heat radiation wavelengths). The transfer matrix of the beam through the layer can be written as:

$$
\begin{aligned}
& {\left[\begin{array}{cc}
A & i B \\
i C & D
\end{array}\right]=\prod_{m=1}^{N} M_{m}=\prod_{m=1}^{N}\left[\begin{array}{cc}
\cos \delta_{m} & \frac{i}{\mu_{m}} \sin \delta_{m} \\
i \mu_{m} \sin \delta_{m} & \cos \delta_{m}
\end{array}\right]} \\
& \delta_{m}=\frac{2 \pi \cdot n_{m} d_{m} \cos \theta_{m}}{\lambda}
\end{aligned}
$$

For polarization of $\mathrm{P}: \quad \mu_{m}=\frac{n_{m}}{\cos \theta_{m}}$

For polarization of S: $\quad \mu_{m}=n_{m} \cos \theta_{m}$

$T=\frac{4 \mu \cdot \mu_{s}}{\left(\mu A+\mu_{s} D\right)^{2}+\left(\mu_{o} \mu_{s} B+C\right)}$

where $n$ is the refractive index, $\theta$ is the radiation angle, $d$ is the layer thickness, $m$ stands for the layer number and $T$ is the emission power.

The refractive index of substrate is $n_{s}=1.5$. Also the $M g F_{2}$ having $n=1.38$ is used as the layer of low refractive index and $Z n S$ with $n=2.29$ is used as the high refractive index layer. The materials selected for layers have relatively high melting temperatures. They are relatively tough and can not be dissolved in water. In the simulation carried out for 5 layers, a quality factor $Q_{1}=1.59$ could be obtained. As shown in Fig.12a, by repeating the semi-layers, a filter having $Q=Q_{1} Q_{2}=2.53$ was obtained. On the other hand, since a thin metal layer on a transparent dielectric is a good reflector for $I R$ and also is a good transmitter for visible radiation, a direct observation of the target is possible. In Fig.12b the transmission curve for the optical filter is also presented. For the Nd:YAG laser, the transmission coefficient is $99.7 \%$.

An appropriate algorithm is designed to reduce the measurement error. Considering the sampling rate of 20 $\mathrm{Hz}$ and $2 \mathrm{~s}$ for refreshing the display, there will be 40 samples in each display. By omitting the out of range data, the measuring error is considerably reduced. Figure 13 presents the measured data for distance of $1.170 \mathrm{~km}$. For primary data, the average and standard deviation are $1175.23 \mathrm{~m}$ and 114.95 , respectively. After the processing, this data change to $1170.46 \mathrm{~m}$ and 2.92 , respectively. Table 1 shows the summery of characteristics of the designed time-of-flight laser range finder.

As a comparison to the work demonstrated by reference $^{[19]}$, if the clock pulse frequency is chosen to be large (a few $\mathrm{GHz}$ ), the error could be as small as 2-5 $\mathrm{mm}$; however, the maximum range decreases sharply to about $34.5 \mathrm{~m}$. Hence, the designed system is optimum for long distances. In addition, the high repetition rate is one of the advantageous of this system which can be used in tracker or seeker systems ${ }^{[20]}$.

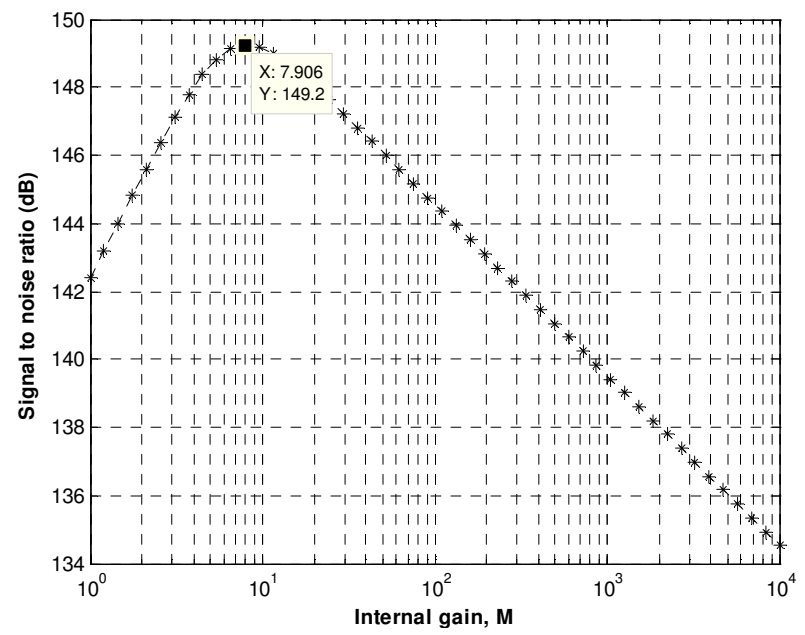

Fig. 10: The signal to noise ratio in terms of $M$

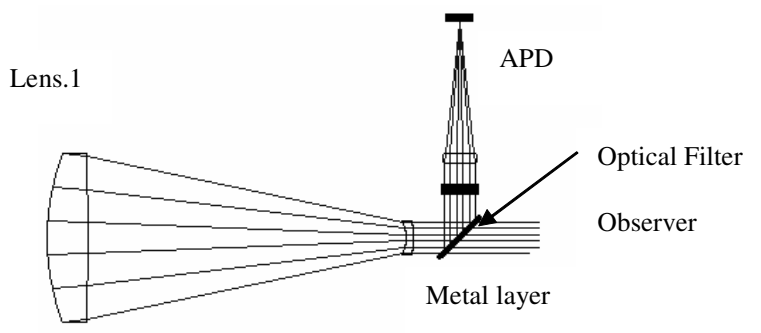

Fig. 11: The designed range finder optics by Zemax software
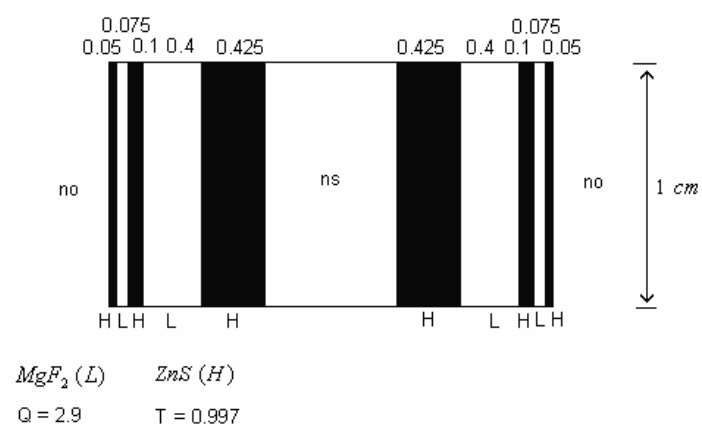

(a) 


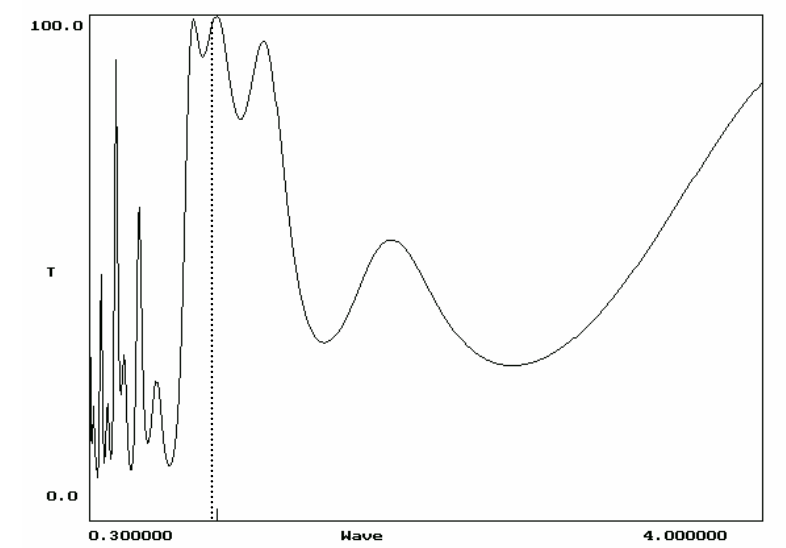

(b)

Fig. 12: (a) The transmission curve and (b) the designed filter from layers of $\mathrm{ZnS}$ and $\mathrm{MgF}_{2}$ with quality factor of 2.53

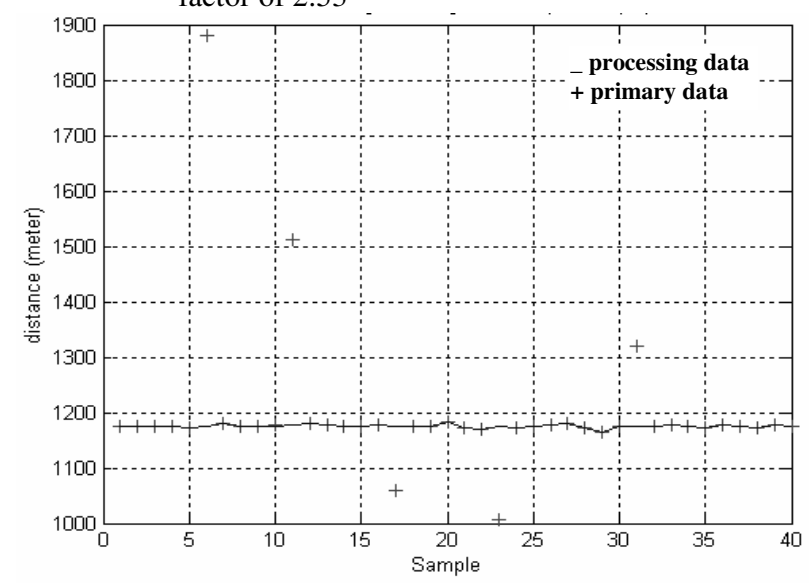

Fig. 13: Measured data for distance of $1.170 \mathrm{~km}$

Table 1: Data representing the time-of-flight laser range finder

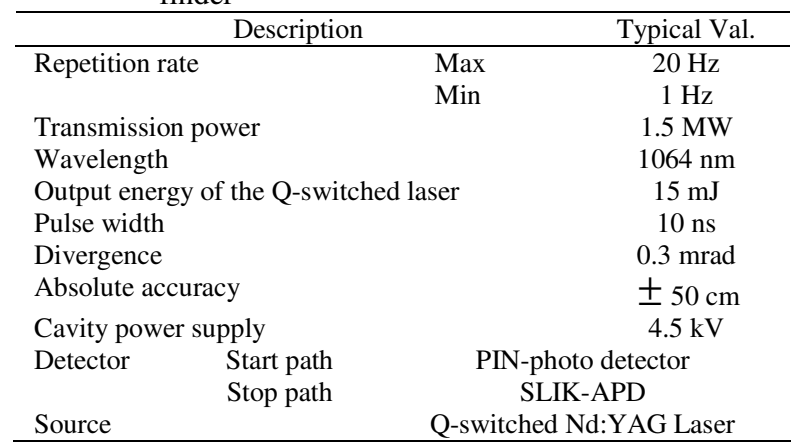

\section{CONCLUSION}

In this paper, design, simulation and implementation of the TOF laser range finder has been presented. A Q-switched Nd:YAG laser having 1.5
MW power with $10 \mathrm{~ns}$ pulse width and repetition rate of $1-20 \mathrm{~Hz}$ was used in the transmitter section. A SLIK APD having responsivity of 18.5-123.1 A/W at 320$351 \mathrm{~V}$ reverse bias voltage, and minimum detectable power of $8.14 \mathrm{nW}$ was used at the receiver. The optical head was designed and simulated by Zemax and its equivalent lenses and filters were used in the practical system. The system was tested frequently in the range of $0.3-20 \mathrm{~km}$. The absolute accuracy of the measured data was $\pm 50 \mathrm{~cm}$ with standard deviation of 2.92 .

\section{REFERENCES}

1. Olyaee, S. and S.M. Nejad, 2007. Nonlinearity and frequency-path modelling of threelongitudinal-mode nanometric displacement measurement system: IET Optoelectron., 1 (5): 211-220.

2. Olyaee, S. and S.M. Nejad, 2007. Characterization of elliptically polarized light and rotation angle of PBS in three-longitudinal-mode laser interferometer using the Jones matrices: J. Appl. Sci., 7 (19): 2806-2811.

3. Zagar, B., 1994. A laser-interferometer measuring displacement with nanometer resolution: IEEE Trans. Inst. measu., 43 (2): 332-336.

4. Martinez, J.C., 2000. A robust photointerferometric technique to obtain the refractive index and thickness of non-absorbing stand-alone films: Meas. Sci. Tech., 11 (8): 1138-1144.

5. Nejad, S.M. and S. Olyaee, 2002. Cross-talk and intermediate frequency deviation effects on phaseshift range finder: J. Sci. Technol., 26 (B4): 647654.

6. Minoni, U. and L. Rovalti, 1999. High performance front-end electronic for frequencymodulated continuous-wave interferometers: IEEE Trans. Inst. Meas., 48 (6): 1191-1196.

7. Fujima, I., S. Iwasaki and K. Seta, 1998. Highresolution distance meter using optical intensity modulation at $28 \mathrm{GHz}$ : Meas. Sci. Tech., 9 (7): 1049-1052.

8. Bazin, G. and B. Journet, 1996. A new laser range-finder based on FMCW-like method: IEEE. Ins. Meas. Technol. Conference, Belgium: 90-93.

9. Barwood, G. and R. Rowley, 1998. High-accuracy length meterology using multiple-stage sweptfrequency interferometry with laser diode: Meas. Sci. Technol., 9 (7): 1036-1041.

10. Kawakami, T and M. Endo, 1994. Adaptive multifrequency modulation method for an advanced laser range finder: IEEE. Trans. Inst. measu., 43 (6): 857-860. 
11. Nejad, S.M. and I. Mohajeri, 2005. Design and simulation of a high resolution FMCW-like laser range finder: Proceedings of ICEE2005: 372-377.

12. Christiet, S., S.L. Hill, B. Buryt, J. Grayt and K.M. Booth, 1995. Design and development of a multi-detecting two-dimensional ranging sensor: Meas. Sci. Technol., 6 (9): 1301-1308.

13. Pellegrini, S., G. Buller, J. Smith, A. Wallace and S. Cova, 2000. Laser-based distance measurement using picosecond resolution time-correlated single-photon counting: Meas. Sci. Technol., 11 (6): 712-716.

14. Jakobsen1, M.L., S. Osten, S. RKitchen, C. DamHansen and S.G. Hanson1, 2003. Multiple-beam time-of-flight sensor based on a vertical cavity surface emitting laser diode array: Meas. Sci. Technol., 14 (3): 329-335.

15. Wei, G.O. and G. Hirzinger, 1997. Active selfcalibration of hand-mounted laser range finders: Proceedings of IEEE: 2789-2794.
16. Refaat, T.F., G.E., Halama and R.J., DeYoung, 2000. Characterization of advanced avalanche photodiode for water vapor lidar receivers: NASA/TP2000, July 2000, pp. 5-18.

17. Refaat, T.F., S.L. William and R.J. DeYoung, 1999. Temperature control of avalanche photodiode using thermoelectric cooler: NASA/TM1999, October 1999, pp. 5-8.

18. Refaat, T.F., G.E., Halama and R.J., DeYoung, 2000. Comparison between super low ionization ratio and reach through avalanche photodiode structures: Opt. Eng., 39 (10): 2642-2650.

19. Kilpela, A., 2004. Pulsed time-of-flight laser range finder techniques for fast, high precision measurement applications: Oulu university press, OULU, pp. 83-86.

20. Makynen, A., 2000. Position-sensitive devices and sensor systems for optical tracking and displacement sensing applications, Oulu University press, OULU, pp. 38-40. 